\title{
Depth, Highness and DNR Degrees ${ }^{\star}$
}

\author{
Philippe Moser ${ }^{1}$ and Frank Stephan ${ }^{2}$ \\ 1 Department of Computer Science, National University of Ireland \\ Maynooth, Co Kildare, Ireland \\ pmoser@cs.nuim.ie \\ 2 Department of Mathematics, The National University of Singapore \\ 10 Lower Kent Ridge Drive, S17, Singapore 119076, Republic of Singapore \\ fstephan@comp.nus.edu.sg
}

\begin{abstract}
A sequence is Bennett deep [5] if every recursive approximation of the Kolmogorov complexity of its initial segments from above satisfies that the difference between the approximation and the actual value of the Kolmogorov complexity of the initial segments dominates every constant function. We study for different lower bounds $r$ of this difference between approximation and actual value of the initial segment complexity, which properties the corresponding $r(n)$-deep sets have. We prove that for $r(n)=\varepsilon n$, depth coincides with highness on the Turing degrees. For smaller choices of $r$, i.e., $r$ is any recursive order function, we show that depth implies either highness or diagonally-non-recursiveness (DNR). In particular, for left-r.e. sets, order depth already implies highness. As a corollary, we obtain that weakly-useful sets are either high or DNR. We prove that not all deep sets are high by constructing a low order-deep set.

Bennett's depth is defined using prefix-free Kolmogorov complexity. We show that if one replaces prefix-free by plain Kolmogorov complexity in Bennett's depth definition, one obtains a notion which no longer satisfies the slow growth law (which stipulates that no shallow set truth-table computes a deep set); however, under this notion, random sets are not deep (at the unbounded recursive order magnitude). We improve Bennett's result that recursive sets are shallow by proving all $K$-trivial sets are shallow; our result is close to optimal.

For Bennett's depth, the magnitude of compression improvement has to be achieved almost everywhere on the set. Bennett observed that relaxing to infinitely often is meaningless because every recursive set is infinitely often deep. We propose an alternative infinitely often depth notion that doesn't suffer this limitation (called i.o. depth). We show that every hyperimmune degree contains a i.o. deep set of magnitude $\varepsilon n$, and construct a $\Pi_{1}^{0}$-class where every member is an i.o. deep set of magnitude $\varepsilon n$. We prove that every non-recursive, non-DNR hyperimmune-free set is i.o. deep of constant magnitude, and that every nonrecursive many-one degree contains such a set.
\end{abstract}

\footnotetext{
* P. Moser was on Sabbatical Leave to the National University of Singapore, supported in part by SFI Stokes Professorship
} and Lectureship Programme. F. Stephan was supported in part by NUS grants R146-000-181-112 and R146-000-184-112. 


\section{Introduction}

The concept of logical depth was introduced by C. Bennett [5] to differentiate useful information (such as DNA) from the rest, with the key observation that non-useful information pertains in both very simple structures (for example, a crystal) and completely unstructured data (for example, a random sequence, a gas). Bennett calls data containing useful information logically deep data, whereas both trivial structures and fully random data are called shallow.

The notion of useful information (as defined by logical depth) strongly contrasts with classical information theory, which views random data as having high information content. I.e., according to classical information theory, a random noise signal contains maximal information, whereas from the logical depth point of view, such a signal contains very little useful information.

Bennett's logical depth notion is based on Kolmogorov complexity. Intuitively a logically deep sequence (or equivalently a set) is one for which the more time a compressor is given, the better it can compress the sequence. For example, both on trivial and random sequences, even when given more time, a compressor cannot achieve a better compression ratio. Hence trivial and random sequences are not logically deep.

Several variants of logical depth have been studied in the past $[1,7,11,12,15]$.

As shown in [15], all depth notions proposed so far can be interpreted in the compression framework which says a sequence is deep if given (arbitrarily) more than $t(n)$ time steps, a compressor can compress the sequence $r(n)$ more bits than if given at most $t(n)$ time steps only. By considering different time bound families for $t(n)$ (e.g. recursive, polynomial time etc.) and the magnitude of compression improvement $r(n)$ - for short: the depth magnitude - (e.g. $O(1), O(\log n))$ one can capture all existing depth notions $[1,7,11,12,15]$ in the compression framework [15]. E.g. Bennett's notion is obtained by considering all recursive time bounds $t$ and a constant depth magnitude, i.e., $r(n)=O(1)$. Several authors studied variants of Bennett's notion, by considering different time bounds and/or different depth magnitude from Bennett's original notion $[1,2,7,11,15]$.

In this paper, we study the consequences these changes of different parameters in Bennett's depth notion entail, by investigating the computational power of the deep sets yielded by each of these depth variants.

- We found out that the choice of the depth magnitude has consequences on the computational power of the corresponding deep sets. The fact that computational power implies Bennett depth was noticed in [11], where it was shown that every high degree contains a Bennett deep set (a set is high if, when given as an oracle, its halting problem is at least as powerful as the halting problem relative to the halting problem: $A$ is high iff $\left.A^{\prime} \geq_{T} \emptyset^{\prime \prime}\right)$. We show that the converse also holds, i.e., that depth implies computational power, by proving that if the depth magnitude is chosen to be "large" (i.e., $r(n)=\varepsilon n$ ), then depth coincides with highness (on the Turing degrees), i.e., a Turing degree is high iff it contains a deep set of magnitude $r(n)=\varepsilon n$.

- For smaller choices of $r$, for example, if $r$ is any recursive order function, depth still retains some computational power: we show that depth implies either highness or diagonally-nonrecursiveness, denoted DNR (a total function is DNR if its image on input $e$ is different from 
the output of the $e$-th Turing machine on input $e$ ). This implies that if we restrict ourselves to left-r.e. sets, recursive order depth already implies highness. We also show that highness is not necessary by constructing a low order-deep set (a set is low if it is not powerful when given as an oracle).

- As a corollary, our results imply that weakly-useful sets introduced in [11] are either high or DNR (set $S$ is weakly-useful if the class of sets reducible to it within a fixed time bound $s$ does not have measure zero within the class of recursive sets).

- Bennett's depth [5] is defined using prefix-free Kolmogorov complexity. Two key properties of Bennett's notion are the so-called slow growth law, which stipulates that no shallow set can quickly (truth-table) compute a deep set, and the fact that neither Martin-Löf random nor recursive sets are deep. It is natural to ask whether replacing prefix-free with plain complexity in Bennett's formulation yields a meaningful depth notion. We call this notion plain-depth. We show that the random is not deep paradigm also holds in the setting of plain-depth. On the other hand we show that the slow growth law fails for plain-depth: every many-one degree contains a set which is not plain-deep of magnitude $O(1)$.

- A key property of depth is that "easy" sets should not be deep. Bennett [5] showed that no recursive set is deep. We improve this result by showing that no $K$-trivial set is deep (a set is $K$-trivial if the complexity of its prefixes is as low as possible). We observe that our result is close to optimal, since there exist deep ultracompressible sets [12].

- In most depth notions, the depth magnitude has to be achieved almost everywhere on the set. Some feasible depth notions also considered an infinitely often version [7]. Bennett noticed in [5] that infinitely often depth is meaningless because every recursive set is infinitely often deep. We propose an alternative infinitely often depth notion that doesn't suffer this limitation (called i.o. depth). We show that little computational power is needed to compute i.o. depth, i.e., every hyperimmune degree contains an i.o. deep set of magnitude $\varepsilon n$ (a set is hyperimmune if it computes a function that is not bounded almost everywhere by any recursive function), and construct a $\Pi_{1}^{0}$-class where every member is an i.o. deep set of magnitude $\varepsilon n$.

For hyperimmune-free sets we prove that every non-recursive, non-DNR hyperimmune-free set is i.o. deep of constant magnitude, and that every nonrecursive many-one degree contains such a set.

In summary, our results show that the choice of the magnitude for logical depth has consequences on the computational power of the corresponding deep sets, and that "larger" depth magnitude does not necessarily equate with "better". We conclude with a few open questions regarding the constant magnitude case.

\section{Preliminaries}

We use standard computability/algorithmic randomness theory notations see $[8,16,18]$. We use $\leq^{+}$to denote less or equal up to a constant term. We fix a recursive 1-1 pairing function $\langle\cdot\rangle: \mathbb{N} \times \mathbb{N} \rightarrow \mathbb{N}$. We use sets and their characteristic sequences interchangeably, we denote the 
binary strings of length $n$ by $2^{n}$, and $2^{\omega}$ denotes the set of all infinite binary sequences. The join of two sets $A, B$ is the set $A \oplus B$ whose characteristic sequence is $A(0) B(0) A(1) B(1) \ldots$, that is, $(A \oplus B)(2 n)=A(n)$ and $(A \oplus B)(2 n+1)=B(n)$ for all $n$. An order function is an unbounded non-decreasing function from $\mathbb{N}$ to $\mathbb{N}$. A time bound function is a recursive order $t$ such that there exists a Turing machine $\Phi$ such that for every $n, \Phi(n)[t(n)] \downarrow=t(n)$, i.e., $\Phi(n)$ outputs the value $t(n)$ within $t(n)$ steps of computation. Set $A$ is left-r.e. iff the set of dyadic rationals strictly below the real number 0.A (a.k.a. the left-cut of $A$ denoted $L(A)$ ) is recursively enumerable (r.e.), i.e., there is a recursive sequence of non-decreasing rationals whose limit is 0.A. All r.e. sets are left-r.e., but the converse fails.

We consider standard Turing reductions $\leq_{T}$, truth-table reductions $\leq_{t t}$ (where all queries are made in advance and the reduction is total on all oracles) and many-one reductions $\leq_{m}$. Two sets $A, B$ are Turing equivalent $\left(A \equiv_{T} B\right)$ if $A \leq_{T} B$ and $B \leq_{T} A$. The Turing degree of a set $A$ is the set of sets Turing equivalent to $A$. Fix a standard enumeration of all oracle Turing machines $\Phi_{1}, \Phi_{2}, \ldots$ The jump $A^{\prime}$ of a set $A$ is the halting problem relative to $A$, i.e., $A^{\prime}=\left\{e: \Phi_{e}^{A}(e) \downarrow\right\}$. The halting problem is denoted $\emptyset^{\prime}$. A set $A$ is high (that is, has high Turing degree) if its halting problem is as powerful as the halting problem of the halting problem, i.e., $\emptyset^{\prime \prime} \leq_{T} A^{\prime}$. High sets are equivalent to sets that compute dominating functions (i.e., sets $A$ such that there is a function $f$ with $f \leq_{T} A$ such that for every computable function $g$ and for almost every $n, f(n) \geq g(n)$ ), i.e., a set is high iff it computes a dominating function [18]. A set $A$ is low if its halting problem is not more powerful than the halting problem of a recursive set, i.e., $A^{\prime} \leq_{T} \emptyset^{\prime}$. Note that $\emptyset^{\prime}$ is high relative to every low set.

If one weakens the dominating property of high sets to an infinitely often condition, one obtains hyperimmune sets. A set is hyperimmune if it computes a function that dominates every recursive function on infinitely many inputs. Otherwise the set is called hyperimmune-free.

Another characterization of computational power used in computability theory is the concept of diagonally non-recursive function (DNR). A total function $g$ is DNR if for every $e, g(e) \neq \Phi_{e}(e)$, i.e., $g$ can avoid the output of every Turing machine on at least one specified input. It is known that every r.e. DNR set is high, actually even Turing equivalent to $\emptyset^{\prime}$. [18].

If one requires the DNR function to be Boolean, one obtains the PA-complete degrees: A degree is PA-complete iff it computes a Boolean DNR function. It is known that there exists low PA-complete degrees [18].

Fix a universal prefix free Turing machine $U$, i.e., such that no halting program of $U$ is a prefix of another halting program. The prefix-free Kolmogorov complexity of string $x$, denoted $K(x)$, is the length of the length-lexicographically first program $x^{*}$ such that $U$ on input $x^{*}$ outputs $x$. It can be shown that the value of $K(x)$ does not depend on the choice of $U$ up to an additive constant. $K(x, y)$ is the length of a shortest program that outputs the pair $(x, y)$, and $K(x \mid y)$ is the length of a shortest program such that $U$ outputs $x$ when given $y$ as an advice. We also consider standard time bounded Kolmogorov complexity. Given time bound $t$ (resp. $s \in \mathbb{N}$ ), $K^{t}(x)$ (resp. $K_{s}(x)$ ) denotes the length of the shortest prefix free program $p$ such that $U(p)$ outputs $x$ within $t(|x|)$ (resp. $s$ ) steps. Replacing $U$ above with a plain (i.e., non prefix- 
free) universal Turing machine yields the notion of plain Kolmogorov complexity, and is denoted $C(x)$. We need the following counting theorem.

Theorem 1. There exists $c \in \mathbb{N}$ such that for every $r, n \in \mathbb{N}, \mid\left\{\sigma \in 2^{n}: K(\sigma) \leq n+K(n)-\right.$ $r\} \mid \leq 2^{n-r+c}$.

A set $A$ is Martin-Löf random (MLR) if none of its prefixes are compressible by more than a constant term, i.e., $\forall n K(A\lceil n) \geq n-c$ for some constant $c$, where $A\lceil n$ denotes the first $n$ bits of the characteristic function of $A$. A set $A$ is $K$-trivial if its complexity is as low as possible, i.e., $\forall n K(A \uparrow n) \leq K(n)+O(1)$. See [13] for more on $C$ and $K$-complexity, MLR and trivial sets.

Effective closed sets are captured by $\Pi_{0}^{1}$-classes. A $\Pi_{1}^{0}$-class $P$ is a class of sequences such that there is a computable relation $R$ such that $P=\left\{S \in 2^{\omega} \mid \forall n R(S\lceil n)\}\right.$.

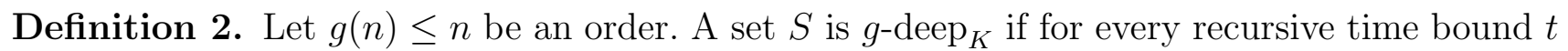
and for almost all $n \in \mathbb{N}, K^{t}(S \uparrow n)-K(S \uparrow n) \geq g(n)$.

A set $S$ is $O(1)$-deep $_{K}($ resp. order-deep $K)$ if it is $c$-deep $K($ resp. $g$-deep $K$ ) for every $c \in \mathbb{N}($ resp. for some recursive order $g$ ). A set is said Bennett deep if it is $O(1)$-deep ${ }_{K}$. We denote by $g$-deep ${ }_{C}$ the above notions with $K$ replaced with $C$. It is easy to see that for every two orders $f, g$ such that $\forall n \in \mathbb{N} f(n) \leq g(n)$, every $g$-deep det is also $f$-deep $_{K}$.

Bennett's slow growth law (SGL) states that creating depth requires time beyond a "recursive amount", i.e., no shallow set quickly computes a deep one.

Lemma 3 (Bennett [5]; Juedes, Lathrop and Lutz [11]). Let $h$ be a recursive order, and

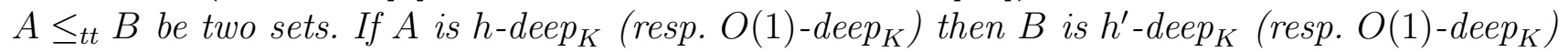
for some recursive order $h^{\prime}$. Furthermore given indices for the truth-table reduction and for $h$, one can effectively compute an index for $h^{\prime}$.

The symmetry of information holds in the resource bounded case.

Lemma 4 (Li and Vitányi [13]). For all time bound there is a time bound $t^{\prime}$ such that for every strings $x, y$ with $|y| \leq t(|x|)$, we have $C^{t}(x, y) \geq C^{t^{\prime}}(x)+C^{t^{\prime}}(y \mid x)-O\left(\log C^{t^{\prime}}(x, y)\right)$. Furthermore given an index for $t$ one can effectively compute an index for $t^{\prime}$.

Corollary 5. Let $t$ be a time bound and $x$, a be strings. Then there exists a time bound $t^{\prime}$ such that for every prefix $y$ of $x$ we have $C^{t}(y \mid a) \geq^{+} C^{t^{\prime}}(x \mid a)-|x|+|y|-O\left(\log C^{t}(y \mid a)\right)$. Furthermore given an index for $t$ one can effectively compute an index for $t^{\prime}$.

Proof. Given $p$ a $C^{t}$-minimal program with advice $a$ for $y,|x|-|y|$ remaining bits, and a delimiter after $p$, one can reconstruct $x$ in time $t^{\prime}(\cdot)=t(\cdot)+O(n)$ steps given a. Thus $C^{t}(y \mid a)+|x|-$ $|y|+O\left(\log C^{t}(y \mid a)\right) \geq^{+} C^{t^{\prime}}(x \mid a)$. 


\section{$3 \quad C$-Depth}

Bennett's original formulation [5] is based on $K$-complexity. In this section we investigate the depth notion obtained by replacing $K$ with $C$, which we call plain depth. We study the interactions of plain depth with the notions of Martin-Löf random sets, many-one degrees and the Turing degrees of deep sets.

\subsection{MLR is not order-deep ${ }_{C}$}

The following result is the plain complexity version of Bennett's result that no MLR sets are Bennett deep.

Theorem 6. For every $M L R A$ and for every recursive order $h, A$ is not $h$-deep . $_{\text {. }}$

Proof. Suppose by contradiction that set $A$ is MLR and $h$-deep $C$, for some $h$ as above. We claim that $\exists^{\infty} n C(A \uparrow n) \geq n-h(n) / 2$. To prove the claim, let $N=\{n \in \mathbb{N}: h(n) \neq h(n-1)\}$. Then given $a=h(n)$ with $n \in N$, the program $p$ : "Print the smallest $m$ such that $h(m)=a$." is a program for $n$ of size $K(a)+O(1)$, i.e.,

$$
K(n) \leq K(a)+O(1) \leq 2 \log h(n)+O(1)<h(n) / 4 .
$$

Suppose $q$ is a $C$-minimal program for $A\lceil n$ of size $n-m$, then appending $2 \log m+K(n)$ bits to $q$ yields a prefix free program $q^{\prime}$ for $A \uparrow n$ of size $2 \log m+K(n)+n-m$.

Since $A$ is MLR we have $\left|q^{\prime}\right| \geq n-O(1)$, i.e., $2 \log m+K(n)+n-m>n-O(1)$ which implies $K(n)>m-2 \log m-O(1)>2 / 3 m$. Let $n \in N$, then $2 / 3 m<K(n)<h(n) / 4$, thus $m<h(n) / 2$, i.e., $C(A\lceil n)>n-h(n) / 2$, which proves the claim.

Since for all $n \in \mathbb{N}$ we have $C^{n^{2}}(A \uparrow n) \leq n+O(1)$ (via a "print" program), it follows that for every $n \in N$,

$$
C^{n^{2}}\left(A\lceil n)-C(A \uparrow n) \leq n+O(1)-n+h(n) / 2 \leq^{+} h(n) / 2\right.
$$

which contradicts that $A$ is $h$-deep ${ }_{C}$.

Sequences that are MLR relative to the halting problem are called 2-random. Equivalently a sequence $A$ is 2-random iff there is a constant $c$ such that $C(A \uparrow n) \geq n-c$ for infinitely many $n[14,17]$. Since there is a constant $c^{\prime}$ such that $n+c^{\prime}$ is a trivial upper bound on the plain Kolmogorov complexity of any string of length $n$, it is clear that no 2-random sequence can be $O(1)$ deep $_{C}$. Thus most MLR sequences are not $O(1)-\operatorname{deep}_{C}$.

\subsection{The SGL fails for $C$-depth}

The following result shows that the Slow Growth Law fails for plain depth.

Theorem 7. Every many-one degree contains a set which is not $O(1)$-deep . 
Proof. Given $A$ different from $\mathbb{N}$, let $B=\left\{2^{2^{p}}: p \in A\right\}$. Given any $k$, choose $m=A(0)+2 A(1)+$ $\ldots+2^{k} A(k)$ and let $n$ be any number between $2^{m}$ and $2^{m+1}$ which has $C$-complexity $m$. Now on one hand $C\left(B\lceil n) \geq \geq^{+} C(n) \geq m\right.$ and on the other hand, one can compute $m$ from the $m$-digit binary number representing $n$ and one can compute $A(0), A(1), \ldots, A(k)$ from $m$ and using $B\left(2^{2^{p}}\right)=A(p)$ one can compute $B \backslash n$ from the binary representation of $n$ and its length $m$ so that $C^{t}(B\lceil n) \leq m+c$ for some time bound $t$ and some constant $c$ independent of $n$ and $m$. This shows that $A$ is not $O(1)$-deep $_{C}$. Clearly $A \leq_{m} B$. Furthermore, $B \leq_{m} A$ by mapping all values of form $2^{2^{p}}$ to $p$ and all other values to a fixed non-element of $A$.

Note that this result shows that order-deep ${ }_{K}$ does not imply order-deep ${ }_{C}$ : all the sets in the truth-table degree of any order-deep ${ }_{K}$ set are all order-deep ${ }_{K}$ (by the SGL), but this degree contains a non order-deep ${ }_{C}$ set by the previous result.

\subsection{Depth implies highness or DNR}

The following result shows that being constant deep for $C$ implies computational power.

Theorem 8. Let $A$ be a $O(1)-$ deep $_{C}$ set. Then $A$ is high or DNR.

Proof. We prove the contrapositive. Suppose that $A$ is neither DNR nor high. Let $f(m)$ be (a coding of $A\left\lceil 2^{m+1}\right.$. Because $f \leq_{t t} A$, there are infinitely many $m$ where $\Phi_{m}(m)$ is defined and equal to $f(m)$. Hence there is an $A$-recursive increasing function $g$ such that, for almost every $m, g(m)$ is the time to find an $m^{\prime} \geq m$ with $A(0) A(1) \ldots A\left(2^{m^{\prime}+1}\right)=\Phi_{m^{\prime}}\left(m^{\prime}\right)$ and to evaluate the expression $\Phi_{m^{\prime}}\left(m^{\prime}\right)$ to verify the finding. As $A$ is not high, there is a recursive increasing function $h$ with $h(m) \geq g(m)$ for infinitely many $m$. Now consider any $m$ where $h(m) \geq g(m)$. Then for the $m^{\prime}$ found for this $m$, it holds that $h\left(m^{\prime}\right) \geq h(m)$ and $h\left(m^{\prime}\right)$ is also larger than the time to evaluate $\Phi_{m^{\prime}}\left(m^{\prime}\right)$. Hence $h(m)$ is larger than the time to evaluate $\Phi_{m}(m)$ for infinitely many $m$ where $\Phi_{m}(m)$ codes $A \uparrow 2^{m+1}$.

For each such $m$, let $n$ be a number with $2^{m} \leq n \leq 2^{m+1}$ and $C(n) \geq m$. Starting with a binary description of such an $n$, one can compute $m$ from $n$ and run $\Phi_{m}(m)$ for $h(m)$ steps and, in the case that this terminates with a string $\sigma$ of length $2^{m+1}$, output $\sigma\lceil n$. It follows from this algorithm that there is a resource-bounded approximation to $C$ such that there exist infinitely many $n$ such that, on one hand $C(A\lceil n) \geq \log (n)$ while on the other hand $A\lceil n$ can be described in $\log (n)+c$ bits using this resource bounded description. Hence $A$ is not $O(1)$-deep . $^{-}$

Since there exists high non-DNR sets, the following result shows DNR is not always the case.

Theorem 9. There exists a set $A$ such that $A$ is $(1-\varepsilon) n$-deep (for any $\varepsilon>0$ ) but $A$ is not $D N R$.

Proof. There is a degree which is high but not DNR [18]. Thus we can, by Theorem 10, select a

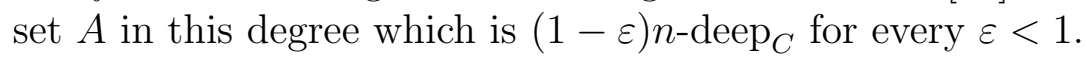




\section{$4 \quad K$-Depth}

Bennett's original depth notion is based on prefix free complexity. He made important connections between depth and truth-table degrees; In particular he proved that the $O(1)$-deep $_{K}$ sets are closed upward under truth-table reducibility, which he called the slow growth law. In the following section we pursue Bennett's investigation by studying the Turing degrees of deep sets. In the first subsection, we investigate the connections between linear depth and high Turing degrees. We then look at the opposite end by studying the interactions of various lowness notions with logical depth.

\subsection{Highness and depth coincide}

The following result shows that at depth magnitude $\varepsilon n$, depth and highness coincide on the Turing degrees. The result holds for both $K$ and $C$ depth.

Theorem 10. For every set $A$ the following statements are equivalent:

1. The degree of $A$ is $\varepsilon n$-deep ${ }_{C}$ for some $\varepsilon>0$.

2. The degree of $A$ is $(1-\varepsilon) n$-deep $p_{C}$ for every $\varepsilon>0$.

3. A is high.

Proof. We prove $(1) \Rightarrow(3)$ using the contrapositive: Let $\varepsilon>0$ and $l \in \mathbb{N}$ such that $\delta<\varepsilon / 3$ with $\delta:=1 / l$. Let $k$ be the limit inferior of the set $\{0,1, \ldots, l\}$ such that there are infinitely many $n$ with $C(A\lceil n) \leq n \cdot k \cdot \delta$. Now one can define, relative to $A$, an $A$-recursive function $g$ such that for each $n$ there is an $m$ with $n \leq m \leq g(n)$ and $C_{g(n)}(A\lceil m) \leq m \cdot k \cdot \delta$. As $A$ is not high, there is a recursive function $h$ with $h(n)>g(n)$ for infinitely many $n$; furthermore, $h(n) \leq h(n+1)$ for all $n$. It follows that there are infinitely many $n$ with $C_{h(n)}(A\lceil n) \leq n \cdot k \cdot \delta$ which is also at most $n \cdot \delta$ away from the optimal value, hence $A$ is not $\varepsilon \cdot n$ deep, which ends this direction's proof.

Let us show (3) $\Rightarrow(2)$. Let $\varepsilon>0, A$ be high, and let $g \leq_{T} A$ be dominating. We construct $B \equiv_{T} A$ such that $B$ is $(1-\varepsilon) n$ - deep $_{C}$.

By definition, if $t$ is a time bound and $i$ an index of $t$ then for every $m \in \mathbb{N} \Phi_{i}(m)[t(m)] \downarrow=$ $t(m)$. Since $g$ is dominating, we have for almost every $m \in \mathbb{N}, t(m)=\Phi_{i}(m)[g(m)] \downarrow$.

We can thus use $g$ to encode all time bounds that are total on all strings of length less or equal to $2^{n}$, into a set $H$, where

$$
H(\langle i, j\rangle)=1 \text { iff } \Phi_{i}(m)\left[g\left(2^{j}\right)\right] \downarrow \text { for all } m \in\left\{1,2, \ldots, 2^{j}\right\} .
$$

Thus $t$ is a (total) time bound iff for almost every $j, H(\langle i, j\rangle)=1$ (where $i$ is an index for $t$ ).

We have $H \leq_{T} A$ and we choose the pairing function $\langle\cdot\rangle$ such that $H\left\lceil n^{2}+1\right.$ encodes the values

$$
\{H(\langle i, j\rangle): i, j \leq n\} .
$$

Let $n \in \mathbb{N}$ and suppose $B \nmid 2^{n}$ is already constructed. Given $A \uparrow n+1$ and $H \uparrow n^{2}+1$, we construct $B \nmid 2^{n+1}$. From $H\left\lceil n^{2}+1\right.$, we can compute the set $L_{n}=\{i \leq n: H(\langle i, n\rangle)=1\}$, i.e., 
a list eventually containing all time bounds that are total on strings of lengths less or equal to $2^{n}$. Let

$$
T_{n}:=\max \left\{\Phi_{i}(m): i \in L_{n}, m \leq 2^{n}\right\} .
$$

Find the lex first string $x_{n}$ of length $2^{n}-1$ such that

$$
C_{T_{n}}\left(x_{n} \mid\left(B \nmid 2^{n}\right) A(n)\right) \geq 2^{n} .
$$

Let $B \uparrow\left[2^{n}, 2^{n+1}-1\right]:=A(n) x_{n}$. By construction we have $B \equiv_{T} A$. Also, $C\left(B \uparrow 2^{n+1} \mid H \uparrow\right.$ $n^{2}+1, A\lceil n+1) \leq+C(n)$, i.e., $C\left(B\left\lceil 2^{n+1}\right) \leq 2 n^{2}\right.$.

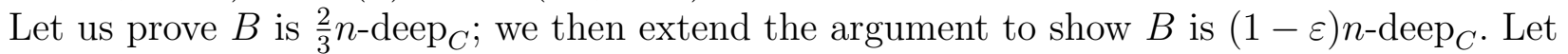
$t$ be a time bound. Let $n$ be large enough such that $t_{1}, t_{2}, t_{3} \in L_{n-2}$ and $t_{1}^{\prime}, t_{2}^{\prime}, t_{3}^{\prime}, t_{4}^{\prime} \in L_{n}$ where the $t_{i}$ 's are derived from $t$ as described below.

Let $j$ be such that $2^{n}<j \leq 2^{n+1}$ and $j^{\prime}=j-\left(2^{n}-1\right)$, i.e., $B\left\lceil j\right.$ ends with the first $j^{\prime}-2$ bits of $x_{n}$ (One bit is "lost" due to the first bit used to encode $A(n)$ ).

We consider two cases, first suppose $j^{\prime}<\log n$. Let $t_{1}$ be a time bound (obtained from $t$ ) such that $C^{t}(B \nmid j) \geq^{+} C^{t_{1}}\left(x_{n-1}, B \nmid 2^{n-1}\right)$, where neither the constant nor $t_{1}$ depends on $j, n$. Let $t_{2}$ be derived from $t_{1}$ using Lemma 4 . We have

$$
\begin{aligned}
& C^{t_{1}}\left(x_{n-1}, B \uparrow 2^{n-1}\right) \\
& \geq C^{t_{2}}\left(B \uparrow 2^{n-1}\right)+C^{t_{2}}\left(x_{n-1} \mid B \uparrow 2^{n-1}\right)-O\left(\log 2^{n}\right) \\
& \geq C^{t_{2}}\left(B \uparrow 2^{n-1}\right)+C_{T_{n-1}}\left(x_{n-1} \mid B \uparrow 2^{n-1}\right)-O(n) \\
& \geq C^{t_{2}}\left(B \uparrow 2^{n-1}\right)+2^{n-1}-O(n) \\
& \geq 2^{n-1}+2^{n-2}+C^{t_{3}}\left(B \uparrow 2^{n-2}\right)-O(n) \\
& \geq \frac{3}{4} 2^{n}-O(n)>\frac{2}{3}\left(2^{n}+j^{\prime}+1\right)=\frac{2}{3} j .
\end{aligned}
$$

because $t_{2} \in L_{n-1}$ by definition of $x_{n-1}$ reapplying the argument above

For the second case, suppose $j^{\prime}>\log n$. We have

$$
\begin{aligned}
& C^{t}(B \uparrow j) \geq C^{t_{1}^{\prime}}\left(x_{n} \uparrow j^{\prime}, B \uparrow 2^{n}\right) \\
& \geq C^{t_{2}^{\prime}}\left(x _ { n } \left\lceilj^{\prime} \mid B\left\lceil 2^{n}\right)+C^{t_{2}^{\prime}}\left(B\left\lceil 2^{n}\right)-O(n)\right.\right.\right. \\
& \text { By Lemma } 4 \\
& \geq C^{t_{3}^{\prime}}\left(x_{n} \mid B \uparrow 2^{n}\right)-2^{n}+j^{\prime}+C^{t_{2}^{\prime}}\left(B \uparrow 2^{n}\right)-O(n) \\
& \geq C_{T_{n}}\left(x_{n} \mid B \uparrow 2^{n}\right)-2^{n}+j^{\prime}+C^{t_{2}^{\prime}}\left(B \nmid 2^{n}\right)-O(n) \\
& \geq 2^{n}-2^{n}+j^{\prime}+C^{t_{2}^{\prime}}\left(B \uparrow 2^{n}\right)-O(n) \\
& \geq j^{\prime}+C^{t_{4}^{\prime}}\left(x_{n-1}, B \uparrow 2^{n-1}\right)-O(n) \\
& \geq j^{\prime}+\frac{3}{4} 2^{n}-O(n) \\
& >\frac{2}{3} j
\end{aligned}
$$

Note that each iteration of the argument above yields a $2^{n-k}$ term $(k=1,2,3, \ldots)$, therefore for

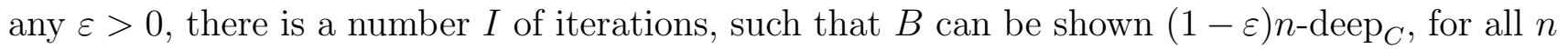
large enough such that $t_{1}, t_{2}, \ldots, t_{3 I} \in L_{n}$. 
Corollary 11. Theorem 10 also holds for K-depth.

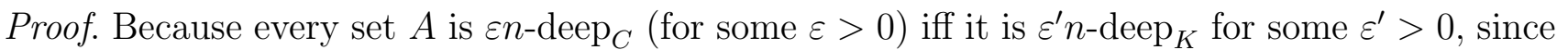
for every $x, C(x) \leq K(x) \leq C(x)+O(\log |x|)$.

\subsection{Depth implies highness or DNR}

An analogue of Theorem 8 holds for $K$.

Theorem 12. Let $A$ be a $h$-deep $p_{K}$ set for some recursive order $h$. Then $A$ is high or DNR.

Proof. We prove the contrapositive. Suppose that $A$ is neither DNR nor high. Let $f(m)$ be (a coding of $A\left\lceil h^{-1}(m)\right.$, where $h^{-1}(m)=\min _{n}\{h(n)=m\}$. The rest of the proof follows the proof of Theorem 8, with $2^{m}$ replaced with $h^{-1}(m)$.

As a corollary, we show that in the left-r.e. case, depth always implies highness.

Corollary 13. If $A$ is left-r.e. and $h$-deep ${ }_{K}$ (for some recursive order $h$ ) then $A$ is high.

Proof. Let $A$ be as above. By definition of $A$ being left-r.e., the left cut $L(A)$ of $A$ is r.e. and $L(A) \equiv_{t t} A$. By Lemma $3, L(A)$ is $h^{\prime}$-deep $_{K}$ (for some recursive order $h^{\prime}$ ). By Theorem 12, $L(A)$ is high or DNR. Since every r.e. DNR set is high, $A$ is high.

As a second corollary, we prove that every weakly-useful set is either high or DNR. A set $A$ is weakly-useful if there is a time-bound $s$ such that the class of all sets truth-table reducible to $A$ with this time bound $s$ is not small, i.e., does not have measure zero within the class of recursive sets; see [11] for a precise definition. In [11], it was shown that every weakly-useful set is $O(1)$-deep $_{K}$ (even order-deep ${ }_{K}$ as observed in [2]) thus generalising the fact that $\emptyset^{\prime}$ is $O(1)$-deep $_{K}$, since $\emptyset^{\prime}$ is weakly-useful.

Theorem 14 (Antunes, Matos, Souto and Vitányi[2]; Juedes, Lathrop and Lutz [11]). Every weakly-useful set is order-deep ${ }_{K}$.

It is shown in [11] that every high degree contains a weakly-useful set. Our results show some type of converse to this fact.

Theorem 15. Every weakly-useful set is either high or DNR.

Proof. This follows from Theorem 12, since every weakly-useful set is order-deep ${ }_{K}$ by Theorem 14. 


\subsection{A low deep set}

We showed in Theorem 10 that every $\varepsilon n$-deep ${ }_{K}$ set is high. Also Theorem 12 shows that every order-deep $_{K}$ set is either high or DNR. Thus one might wonder whether there exists any non-high order-deep $_{K}$ set. We answer this question affirmatively by showing there exist low order-deep ${ }_{K}$ sets.

Theorem 16. If $A$ has $P A$-complete degree, then there exists a weakly-useful set $B \equiv_{T} A$.

Proof. Let $f \leq_{T} A$ be a Boolean DNR function and let $g(n):=1-f(n)$. It follows that if $\Phi_{e}$ is Boolean and total, then $g(e)=\Phi_{e}(e)$. One can thus encode $g$ into a set $B \leq_{T} A$ such that for every $e$ such that $\Phi_{e}$ is Boolean and total and for every $x, B(\langle e+1, x\rangle)=\Phi_{e}(x)$. One can also encode $A$ into $B$ (for example, $B(\langle 0, x\rangle)=A(x))$ so that $A \equiv_{T} B$. Thus for every recursive set $L$ there exists $e$ such that for every string $x$, we have $L(x)=B\left(r_{e}(x)\right)$, where $r_{e}(x)=\langle e, x\rangle$ is computable within $s(n)=n^{2}$ steps (by using a lookup table on small inputs). It follows that every recursive set is truth-table reducible to $B$ within time $s(n)=n^{2}$. Because the class of recursive sets does not have measure zero within the class of recursive sets [11], it follows that $B$ is weakly-useful.

Corollary 17. If $A$ has PA-complete degree, then there exists an order-deep ${ }_{K}$ set $B \equiv_{T} A$. Furthermore, there is a $\Pi_{1}^{0}$-class only consisting of order-deep ${ }_{K}$ sets.

Proof. This follows from Theorems 14 and 16.

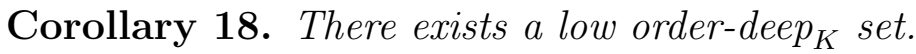

Proof. There exists low sets $A$ of PA-complete degree [18]. By Theorem 17 there exists an order-deep $_{K}$ set $B \equiv_{T} A$. Since $A$ is low it follows that $B$ is low.

Note that this result also implies both the existence of order-deep ${ }_{K}$ sets which are low for $\Omega$, and the existence of order-deep ${ }_{K}$ sets which are nonrecursive and hyperimmune-free, both existence proofs are based on the corresponding basis theorems $[8,10]$. A set $A$ is said low for $\Omega$ iff Chaitin's $\Omega$ is Martin-Löf random relative to $A$. The reason one uses PA-complete sets instead of merely Martin-Löf random sets (which also satisfy all basis theorems), is that Martin-Löf random sets

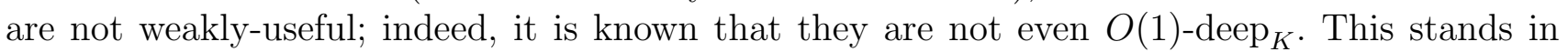
contrast to the following result.

Corollary 19. There are two Martin-Löf random sets $A$ and $B$ such that $A \oplus B$ is order-deep . $_{\text {. }}$

Proof. Barmpalias, Lewis and Ng [4] showed that every PA-complete degree is the join of two Martin-Löf random degrees; hence there are Martin-Löf random sets $A, B$ such that $A \oplus B$ is a hyperimmune-free PA-complete set. Thus, by Theorem 16 there is a weakly-useful set Turing reducible to $A \oplus B$ which, due to the hyperimmune-freeness, is indeed truth-table reducible to $A \oplus B$. It follows that $A \oplus B$ is itself weakly-useful and therefore order-deep ${ }_{K}$ by Corollary 17 . 


\section{$4.4 \quad$ No $K$-trivial is $O(1)-$ deep $_{K}$}

A key property of depth is that "easy" sets should not be deep. Bennett [5] showed that no recursive set is deep. Here we improve this result by showing that no $K$-trivial set is deep. As we will see this result is close to optimal.

Theorem 20. No K-trivial set is $O(1)$-deep ${ }_{K}$.

Proof. Let $A$ be $K$-trivial and $c \in \mathbb{N}$ such that $\forall n \in \mathbb{N}, K(A\lceil n) \leq K(n)+c$. Let $d$ be such that for every string $x, K(x) \geq K(|x|)-d$ and let $g(n)=n^{2}$. There exists a constant $d^{\prime}$ such that the set $M=\left\{n \in \mathbb{N}: K^{g}(n) \leq K(n)+d^{\prime}\right\}$ is infinite (see [8] p. 139). Note that $M$ is co-r.e., i.e., there exists uniformly recursive approximations $M_{1} \supseteq M_{2} \supseteq \ldots \supseteq M$ of $M$. Let $c^{\prime}=\liminf _{n \in M}\left|\left\{\sigma \in 2^{n}: K(\sigma) \leq K^{g}(n)+c\right\}\right|$. By Theorem 1, $c^{\prime}<\infty$. Consider the function

$$
f(n)=\min _{s}\left\{n \notin M_{s} \text { or there exists } c^{\prime} \sigma \in 2^{n} \text { with } K_{s}(\sigma) \leq K^{g}(n)+c\right\} .
$$

By modifying $f$ on the finitely many values before the liminf is reached, $f$ is recursive. Wlog $f$ is bounded by a time bound which we also denote $f$. We have $\exists^{\infty} n \in M$ such that $K^{f}(A \uparrow$ $n) \leq K(n)+c+d^{\prime}$ thus for each of these infinitely many $n$ 's we have $K^{f}(A\lceil n)-K(A\lceil n) \leq$ $K(n)+c+d^{\prime}-K(n)+d=c+d+d^{\prime}$, i.e., $A$ is not $O(1)-$ deep $_{K}$.

Call a set $A$ ultracompressible if for every recursive order $g$ and all $n, K\left(A\lceil n) \leq^{+} K(n)+g(n)\right.$. The following theorem shows that our result is close to optimal.

Theorem 21 (Lathrop and Lutz [12]). There is an ultracompressible set $A$ which is $O(1)$-deep . .

Theorem 22 (Herbert [9]). There is a set $A$ which is not $K$-trivial but which satisfies that for every $\Delta_{2}^{0}$ order $g$ and all $n, K\left(A\lceil n) \leq^{+} K(n)+g(n)\right.$.

It would be interesting to know whether such sets as found by Herbert can be $O(1)-$ deep $_{K}$. The result of Herbert is optimal, Csima and Montalbán [6] showed that such sets do not exist when using $\Delta_{4}^{0}$ orders and Baartse and Barmpalias [3] improved this non-existence to the level $\Delta_{3}^{0}$.

Theorem 23 (Baartse and Barmpalias [3]). There is a $\Delta_{3}^{0}$ order $g$ such that a set $A$ is $K$-trivial iff $K\left(A\lceil n) \leq^{+} K(n)+g(n)\right.$ for all $n$.

That is, the difference between a $K$-trivial and an ultracompressible set is less than the difference of two orders of different complexity. The existence of an ultracompressible $O(1)$-deep ${ }_{K}$ set was proved in [12]. This shows that Theorem 20 is close to optimal.

\section{Infinitely Often Depth and Conditional Depth}

Bennett observed in [5] that being infinitely often Bennett deep is meaningless, because all recursive sets are infinitely often deep. A possibility for a more meaningful notion of infinitely often depth, is to consider a depth notion where the length of the input is given as an advice. We call this notion i.o. depth. 
Definition 24. A set $A$ is i.o. $O(1)$-deep $_{K}$ if for every $c \in \mathbb{N}$ and for every time bound $t \exists^{\infty} n$ such that $K^{t}(A\lceil n \mid n)-K(A\lceil n \mid n) \geq c$.

If we replace $K$ with $C$ in the above definition, we call the corresponding notion i.o. $O(1)$-deep ${ }_{C}$. The fact that all recursive sets are infinitely often deep in Bennett's appraoch does no longer hold for i.o. depth as defined above.

Lemma 25. Let $A$ be recursive. Then $A$ is neither i.o. $O(1)$-deep $p_{C}$ nor i.o. $O(1)$-deep ${ }_{K}$.

Proof. Let $A$ be recursive and $t$ be a time bound. Wlog $A$ is recursive in time $t$, i.e., for every $n \in \mathbb{N}$ we have $C^{t}\left(A\lceil n \mid n) \leq c\right.$ for some constant $c$, thus $\forall n C^{t}(A\lceil n \mid n)-C(A\lceil n \mid n)<c$. The $K$ case is similar.

The following shows that very little computational power is needed to compute an i.o. deep set.

Theorem 26. 1. There is a $\Pi_{1}^{0}$-class such that every member is i.o. $\varepsilon n$-deep ${ }_{C}$ for all $\varepsilon<1$. In particular there is such a set of hyperimmune-free degree. Furthermore, every hyperimmune Turing degree contains such a set.

2. Every nonrecursive many-one degree contains an i.o. $O(1)-$ deep $_{C}$ set.

3. If $A$ is not recursive, not $D N R$ and hyperimmune-free, then $A$ is i.o. $O(1)-$ deep $_{C}$.

Proof. This result is obtained by splitting the natural numbers recursively into intervals $I_{n}=$ $\left\{a_{n}, \ldots, b_{n}\right\}$ such that $b_{n}=\left(2+a_{n}\right)^{2}$. Now one defines the $\Pi_{1}^{0}$-class such that for each $n=$ $\langle e, k\rangle$ where $t=\Phi_{e}$ is defined up to $b_{n}$, a string $\tau \in\{0,1\}^{b_{n}-a_{n}+1}$ is selected such that for all $\sigma \in\{0,1\}^{a_{n}}, C^{t}(\sigma \tau) \geq b_{n}-2 a_{n}-2$ and then it is fixed that all members $A$ of the $\Pi_{1}^{0}-$ class have to satisfy $A(x)=\tau\left(x-a_{n}\right)$ for all $x \in I_{n}$. Since there are $2^{b_{n}-a_{n}+1}$ strings $\tau$ and for each program of size below $b_{n}-2 a_{n}-2$ can witness that only $2^{a_{n}}$ many $\tau$ are violating $C^{t}(\sigma \tau) \geq|\tau|-|\sigma|$ for some $\sigma \in\{0,1\}^{a_{n}}$, there will be less than $2^{b_{n}-a_{n}+1}-2^{b_{n}-a_{n}}$ many $\tau$ that get disqualified and so the search finds such a $\tau$ whenever $\Phi_{e}$ is defined up to $b_{n}$. Hence, for every total $t=\Phi_{e}$, there are infinitely many intervals $I_{n}$ with $n$ of the form $\langle e, k\rangle$ such that on these $I_{n}, C^{t}\left(A(0) A(1) \ldots A\left(b_{n}\right) \mid n\right) \geq C^{t}\left(A(0) A(1) \ldots A\left(b_{n}\right)\right)-\log (n) \geq b_{n}-3 a_{n}$ and $C\left(A(0) A(1) \ldots A\left(b_{n}\right) \mid n\right) \leq a_{n}+c$ for a constant $c$, as the program only needs to know how $A$ behaves below $a_{n}$ and can fill in the values of $\tau$ on $I_{n}$. So the complexity improves after time $t\left(b_{n}\right)$ from $b_{n}-3 a_{n}$ to $a_{n}$ and, to absorb constants, one can conservatively estimate the improvement by $b_{n}-5 a_{n}$. By the choice of $a_{n}, b_{n}$, the ratio $\left(b_{n}-5 a_{n}\right) / b_{n}$ tends to 1 and therefore every $A$ in the $\Pi_{1}^{0}$-class is $\varepsilon n$-deep ${ }_{C}$ for every $\varepsilon<1$. Note that there are hyperimmune-free sets inside this $\Pi_{1}^{0}$-class, as it has only nonrecursive members.

Furthermore, one can see that the proof also can be adjusted to constructing a single set in a hyperimmune Turing degree rather than constructing a full $\Pi_{1}^{0}$-class. In that case one takes some function $f$ in this degree which is not dominated by any recursive function and then one permits for each $n=\langle e, k\rangle$ the time $\Phi_{e}\left(b_{n}\right)$ in the case that $\Phi_{e}\left(b_{n}\right)<f(k)$ and chooses $\tau$ accordingly and one takes $\tau=0^{b_{n}-a_{n}+1}$ in the case that $\Phi_{e}$ does not converge on all values below $b_{n}$ within time $f(k)$ otherwise. This construction is recursive in the given degree and a slight modification of this construction would permit to code the degree into the set $A$. 
For the second item, consider a set $A \subseteq\left\{4^{n}: n \in \mathbb{N}\right\}$. Every many-one degree contains such a set. For each binary string $\sigma$, let

$S_{\sigma}=\left\{\tau \in\{0,1\}^{*}: 4^{|\sigma|-1}<|\tau| \leq 4^{|\sigma|}\right.$ and $\tau\left(4^{n}\right)=\sigma(n)$ for all $n<|\sigma|$ and $\tau(n)=0$ for all $n<|\tau|$ which are not a power of 4$\}$.

In other word, for every $A \subseteq\left\{4^{n}: n \in \mathbb{N}\right\}, S_{A(1) A(4) A(16) \ldots A\left(4^{n}\right)}$ contains those $\tau$ which are a prefix of $A$ and for which $\tau\left(4^{n}\right)$ is defined but not $\tau\left(4^{n+1}\right)$. For each $e, k, n$ where $\Phi_{e}$ is a total function $t$, we now try to find inductively for $m=4^{n}+1,4^{n}+2, \ldots, 4^{n+1}$ strings $\sigma_{m} \in\{0,1\}^{n+1}$ such that whenever $\sigma_{m}$ is found then it is different from all those $\sigma_{m^{\prime}}$ which have been found for some $m^{\prime}<m$ and the unique $\tau \in S_{\sigma_{m}} \cap\{0,1\}^{m}$ satisfies $C^{t}(\tau \mid m) \geq e+3 k$. Note that due to the resource-bound on $C^{t}$ one can for each $m^{\prime}<m$ check whether $\sigma_{m^{\prime}}$ exists and take this information into account when trying to find $\sigma_{m}$. Therefore, for those $m$ where $\sigma_{m}$ exists, the $\tau \in S_{\sigma_{m}} \cap\{0,1\}^{m}$ can be computed from $m, e$ and $k$ and hence $C(\tau \mid m) \leq e+k+c$ for some constant $c$ independent of $e, k, n, m$.

Now assume that $A$ is not infinitely often $O(1)$-deep $_{C}$. Then there is a total function $t=\Phi_{e}$ and a $k>c$ such that $C(\tau|| \tau \mid) \geq C^{t}(\tau|| \tau \mid)-k$ for all prefixes $\tau$ of $A$. It follows that in particular never a $\sigma_{m}$ with $S_{\sigma_{m}}$ consisting of prefixes of $A$ is selected in the above algorithm using $e, k$. This then implies that for almost all $n$ and the majority of the $m$ in the interval from $4^{n}$ to $4^{n+1}$ (which are those for which $\sigma_{m}$ does not get defined) it holds that $C^{t}(\tau \mid m) \leq e+3 k$ for the unique $\tau \in S_{A(1) A(4) A(16) \ldots A\left(4^{n}\right)} \cap\{0,1\}^{m}$. There are at most $2^{e+3 k+2}$ many strings $\sigma \in\{0,1\}^{n+1}$ such that at least half of the members $\tau$ of $S_{\sigma}$ satisfy that $C(\tau|| \tau \mid) \leq e+3 k$ and there is a constant $c^{\prime}$ such that for almost all $n$ the corresponding $\sigma$ satisfy $C(\sigma \mid n) \leq e+3 k+c^{\prime}$. It follows that $C(\tau|| \tau \mid) \leq e+3 k+c^{\prime \prime}$ for some constant $c^{\prime \prime}$ and almost all $n$ and all $\tau \in S_{A(1) A(4) A(16) \ldots A\left(4^{n}\right)}$; in other words, $C(\tau|| \tau \mid) \leq e+3 k+c^{\prime \prime}$ for some constant $c^{\prime \prime}$ and almost all prefixes $\tau$ of $A$. Hence $A$ is recursive [13, Exercise 2.3.4 on page 131].

For the third item, let $A$ be as above, and let $t$ be a time bound. Let $\tilde{h}(n)=\min _{m>n}\left\{C^{t}(A \uparrow\right.$ $m \mid m)>n\}$. Since $\tilde{h} \leq_{T} A$ and $A$ is hyperimmune-free, there exists a recursive $h$ such that $\forall n \in \mathbb{N} h(n)>\tilde{h}(n)$. Wlog we can choose $h$ such that $\forall n \in \mathbb{N} h(n+1)>\tilde{h}(h(n))$. Let $g \leq_{T} A$ be defined by $g(n)=A \uparrow h(n+1)$. Because $A$ is not DNR, we have $\exists^{\infty} n \Phi_{n}(n)=A \uparrow h(n+1)$. Thus, $\forall n \exists m h(n) \leq m<h(n+1)$ and $C^{t}(A \uparrow m \mid m) \geq h(n)$.

Let $e$ be an index of a program such that $\Phi_{e}(m)=\Phi_{n}(n) \uparrow m$ with $n$ satisfying $h(n) \leq m<$ $h(n+1)$. Thus $\exists^{\infty} n \exists m h(n) \leq m<h(n+1)$ and $C^{t}(A\lceil m \mid m) \geq h(n)$ and $C(A \uparrow m \mid m) \leq O(1)$, i.e., $A$ is i.o. $O(1)-\operatorname{deep}_{C}$.

\section{Conclusion}

We conclude that the choice of the depth magnitude has consequences on the computational power of the corresponding deep sets, and that "larger" magnitudes is not necessarily "better". Therefore choosing the appropriate depth magnitude for one's purpose is delicate, as the corresponding depth notions might be very different. When the depth magnitude is large, we proved 
that depth and highness coincide. We showed that this is not the case for smaller depth magnitude by constructing a low order deep set, but the set is not r.e. We therefore ask whether there is a low $O(1)$-deep $_{K}$ r.e. set.

From our results, for magnitudes of order $O(1), K$-depth behaves better than $C$-depth. To further strengthen that observation we ask whether there is an MLR $O(1)$-deep set.

\section{References}

1. Luis Filipe Coelho Antunes, Lance Fortnow, Dieter van Melkebeek and N. Variyam Vinodchandran. Computational depth: Concept and applications. Theoretical Computer Science, 354:391-404, 2006.

2. Luis Filipe Coelho Antunes, Armando Matos, Andre Souto and Paul M. B. Vitányi. Depth as randomness deficiency. Theory of Computing Systems, 45(4):724-739, 2009.

3. Martijn Baartse and George Barmpalias. On the Gap Between Trivial and Nontrivial Initial Segment Prefix-Free Complexity. Theory of Computing Systems, 52(1):28-47, 2013.

4. George Barmpalias, Andy E. M. Lewis and Keng Meng Ng. The importance of $\Pi_{1}^{0}$-classes in effective randomness. The Journal of Symbolic Logic, 75(1):387-400, 2010.

5. Charles H. Bennett. Logical depth and physical complexity. The Universal Turing Machine, A Half-Century Survey, pages 227-257, 1988.

6. B.F. Csima and A. Montalbán. A minimal pair of K-degrees. Proc. of the American Mathematical society, 134:1499-1502, 2006.

7. David Doty and Philippe Moser. Feasible depth. In S. Barry Cooper, Benedikt Löwe, and Andrea Sorbi, editors, Computability in Europe, volume 4497 of Lecture Notes in Computer Science, pages 228-237. Springer, 2007.

8. Rodney G. Downey and Denis R. Hirschfeldt. Algorithmic Randomness and Complexity. Springer, 2010.

9. Ian-Cadoc Herbert. Weak Lowness Notions for Kolmogorov Complexity. University of California at Berkeley, 2013.

10. Carl G. Jockusch and Robert I. Soare. $\Pi_{1}^{0}$ classes and degrees of theories. Transactions of the American Mathematical Society, 173:33-56, 1972.

11. David W. Juedes, James I. Lathrop and Jack H. Lutz. Computational depth and reducibility. Theoretical Computer Science, 132:37-70, 1994.

12. James I. Lathrop and Jack H. Lutz. Recursive computational depth. Information and Computation, 153(1):139-172, 1999.

13. Ming Li and Paul M. B. Vityányi. An Introduction to Kolmogorov Complexity and its Applications. Springer Verlag, New York, 2008.

14. Joseph S. Miller. Every 2-random real is Kolmogorov random. The Journal of Symbolic Logic, 69(3):907-913, 2004.

15. Philippe Moser. On the polynomial depth of various sets of random strings. Theoretical Computer Science, 477:96-108, 2013.

16. André Nies. Computability and Randomness. Oxford University Press, 2009. 
17. André Nies, Sebastiaan A. Terwijn and Frank Stephan. Randomness, relativization and Turing degree. The Journal of Symbolic Logic, 70(2):515-535, 2005.

18. Piergiorgio Odifreddi. Classical Recursion Theory: The Theory of Functions and Sets of Natural Numbers, volume 1. Elsevier, 1989. 